\title{
Physiopathologie de la constipation
}

\author{
Peter J. Milla \\ Unité de Gastro-entérologie et d'Auto-immunité, UCL Institute of Child Health, Londres, Royaume-Uni
}

\begin{abstract}
Mots-clés
Constipation, physiopathologie $\cdot$ Transit colique •

Mécanismes pathogènes, constipation
\end{abstract}

\section{Résumé}

La constipation est un trouble extrêmement fréquent chez l'enfant puisque jusqu'à $25 \%$ des consultations de gastroentérologie et $3 \%$ des visites pédiatriques ambulatoires lui sont imputables. Ce trouble est d'origine fonctionnelle dans $90 \%$ des cas et il n'existe une cause organique que dans seulement $10 \%$ des cas. Les causes fréquentes de constipation de l'enfant sont les suivantes: anomalies intestinales congénitales; affections neurologiques, particulièrement infirmité motrice cérébrale, troubles médullaires et hypotonie; troubles endocrinies et métaboliques (hypothyroïdie, mucoviscidose, hypercalcémie, diabète, acidose rénale) et utilisation de médicaments constipants tels que certains antiacides, le sucralfate, le fer, des produits contenant de la codéine, l'imipramine, la phénytoïne, etc. La plus fréquente des anomalies congénitales est la maladie de Hirschsprung, dont l'incidence est de 1 cas pour 4500 naissances vivantes contre 1 pour 7000 naissances vivantes en ce qui concerne les malformations anorectales et 1 pour 40000 pour ce qui est des autres troubles neuromusculaires intestinaux. Une atteinte organique démontrable est présente chez moins de $5 \%$ des enfants consultant pour la première fois pour d'une constipation.

Copyright $\odot 2007$ Nestec Ltd., Vevey/S. Karger AG, Basel
C 2007 Nestec Ltd., Vevey/S. Karger AG, Basel 0250-9644/07/0652-0055\$23.50/0

Accessible en ligne à: www.karger.com/anf
La constipation est un trouble extrêmement fréquent chez l'enfant puisque jusqu'à $25 \%$ des consultations de gastro-entérologie et $3 \%$ des visites pédiatriques ambulatoires lui sont imputables. Ce trouble est d'origine fonctionnelle dans $90 \%$ des cas et il n'existe une cause organique que dans seulement $10 \%$ des cas. Les causes fréquentes de constipation de l'enfant sont les suivantes anomalies intestinales congénitales; affections neurologiques, particulièrement infirmité motrice cérébrale, troubles médullaires et hypotonie; troubles endocriniens et métaboliques (hypothyroïdie, mucoviscidose, hypercalcémie, diabète, acidose rénale) et utilisation de médicaments constipants tels que certains antiacides, le sucralfate, le fer, des produits contenant de la codéine, l'imipramine, la phénytoïne, etc. La plus fréquente des anomalies congénitales est la maladie de Hirschsprung, dont l'incidence est de 1 cas pour 4500 naissances vivantes contre 1 pour 7000 naissances vivantes en ce qui concerne les malformations anorectales et 1 pour 40000 pour ce qui est des autres troubles musculaires intestinaux. Une atteinte organique démontrable est présente chez moins de $5 \%$ des enfants consultant pour la première fois pour une constipation [1].

$\mathrm{Au}$-delà de la période néonatale, la forme la plus fréquente de constipation est la constipation fonctionnelle, ou constipation idiopathique, mégacôlon idiopathique ou rétention fécale fonctionnelle. Chez $40 \%$ des enfants atteints d'une constipation fonctionnelle, les signes apparaissent au cours de la première année de la vie. Cette pathologie débute habituellement au moment de l'ap-
Peter J. Milla, MD

Gastroenterology Unit, Institute of Child Health

30 Guildford Street

London WC1N 1EH (UK)

Tel. +44 2079052 632, Fax +44 2074391 791, E-Mail pjmilla@onetel.net 
prentissage de l'utilisation des toilettes ou lors de l'entrée à l'école et est plus prévalente chez les garçons que chez les filles [2]. La constipation fonctionnelle de l'enfant diffère significativement de la constipation de l'adulte. Une incontinence fécale est plus fréquente dans la constipation de l'enfant que dans celle de l'adulte et est associée à un comportement de rétention chez l'enfant et d'effort chez l'adulte. La réussite du traitement est plus fréquente chez l'enfant que chez l'adulte.

Nous discuterons ici des mécanismes normaux du transit colique et de la défécation, puis des mécanismes pathogènes connus responsables de constipation.

\section{Transit colique normal et motricité colique}

\section{Transit colique}

Le transit du contenu intestinal se déroule en 36 à 48 heures chez un sujet en bonne santé. Cette durée varie en fonction des régions de l'intestin, puisqu'elle est de quelques secondes ou minutes dans l'œesophage, de $30 \mathrm{minu}-$ tes à 2 heures dans l'estomac et de 1 à 4 heures dans l'intestin grêle, la durée restante étant celle du transit colique. De ce fait, la totalité du transit intestinal donne une bonne indication du temps de transit colique. Le côlon est responsable de la récupération d'eau, de calories et d'électrolytes à partir du contenu intestinal, puis de l'excrétion des substances non digérées. Il est donc avantageux que le temps passé par le contenu endoluminal dans le côlon soit long. Plusieurs facteurs affectent la motricité colique, le temps de transit et la fréquence des défécations. Une alimentation riche en fibres, une personnalité extravertie et le sexe masculin sont corrélés à un temps de transit plus rapide et à une plus grande fréquence des selles [3, 4].

Diverses méthodes ont été utilisées afin de mesurer le transit colique, notamment la scintigraphie des selles ou de l'abdomen ou la mesure du temps de transit d'un marqueur non absorbé à travers la totalité de l'intestin. Cette dernière méthode utilise des marqueurs radio-opaques ou un colorant tel que le rouge carmin, qui peut être facilement détecté dans les selles, et est la plus fréquemment utilisée en pratique clinique. Le rouge carmin mesure le temps de transit de la phase liquide dans l'intestin et les marqueurs radio-opaques celui de la phase solide. La méthode décrite par Metcalf et coll. [5] est la plus utile et différencie facilement une obstruction rectale, due à une rétention fonctionnelle ou à une atteinte altérant la fonction du sphincter anal, d'une constipation par transit lent ou inertie colique. En résumé, dans le cadre de cette méthode, des marqueurs radio-opaques de trois formes différentes sont administrés par voie orale lors de 3 jours consécutifs, une forme différente chaque jour, et une radiographie abdominale sans préparation est effectuée le $4^{\text {ème }}$ jour afin de déterminer la position des différentes formes dans le côlon. Chez les sujets en bonne santé, tous les marqueurs du Jour 1 doivent avoir été expulsés. En utilisant ce type de méthode et des moyens modifiés d'analyse, il est possible de déterminer le temps de transit à travers la totalité du côlon chez l'enfant en bonne santé. Il existe d'importantes variations qui sont similaires chez les enfants plus âgés et les adultes, la durée moyenne étant de 30,7 $\pm 17,5$ heures. Les temps de transit dans le côlon droit, le côlon gauche et le rectosigmoïde ont été respectivement estimés à $8,9 \pm 6,4,8,7 \pm 8,7$ et 13,0 \pm 9,9 heures, au moyen de marqueurs radio-opaques et d'une méthode d'analyse segmentaire. Dans le cas d'une obstruction rectale, tous les marqueurs seront retrouvés dans le rectum et, dans la constipation par transit lent, ils seront répartis dans l'ensemble du côlon (fig. 1).

Le contenu intestinal est propulsé le long du côlon par l'activité motrice (ou motricité) colique. Pendant la plus grande partie du temps, l'activité motrice colique se caractérise par une quiescence ou des contractions segmentaires de basse amplitude qui ne sont pas coordonnées entre les différentes régions coliques [6]. Ces contractions segmentaires mélangent le contenu colique, l'amenant au contact de la muqueuse afin de faciliter l'absorption de l'eau et des nutriments de l'épithélium tels que les acétates et les butyrates. Les contractions segmentaires sont responsables du mouvement du contenu colique dans les deux directions, antérograde et rétrograde, facilitant ainsi l'exposition maximale de ce contenu à la muqueuse.

Plusieurs fois par jour, une onde péristaltique à haute pression apparaît et parcourt une longueur considérable du côlon, propulsant le contenu colique devant elle. Des contractions péristaltiques de haute amplitude (CPHA) ont été observées pour la première fois par Cannon voici plus de 100 ans, lors d'études radiologiques du côlon chez l'animal et l'homme [7]. Les ondes péristaltiques peuvent apparaître à divers sites du côlon, depuis le caecum jusqu'à la partie distale. Il s'agit du principal événement moteur responsable du transport du contenu colique. Lactivité motrice colique a été suivie dans le côlon transverse, descendant et sigmoïde lors d'une étude menée chez 14 volontaires en bonne santé. Les CPHA ont été facilement identifiées par leur amplitude élevée caractéristique de $<200 \mathrm{~mm} \mathrm{Hg}$, leur durée >10 secondes, une propagation sur au moins $30 \mathrm{~cm}$ et une absence de chevauchement avec d'autres contractions [6]. Chez l'adulte, les CPHA apparaissent $4-6$ fois/jour après prise de laxatifs $[8,9]$ et moins 


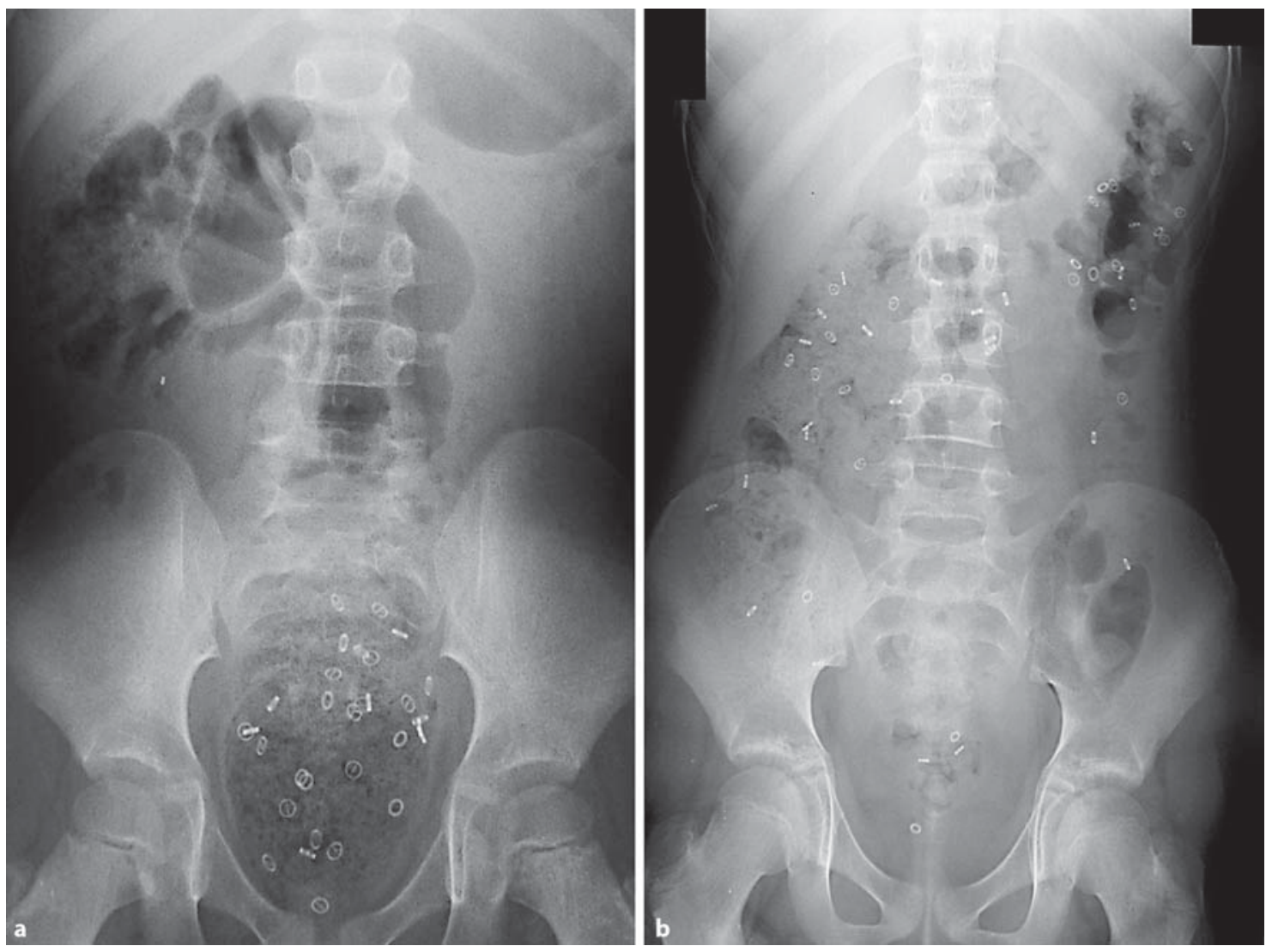

Fig. 1. Radiographie abdominale sans préparation lors d'études du transit par marqueurs radio-opaques. a Rétention rectale montrant l'accumulation des marqueurs dans le rectum. b Constipation par transit lent avec répartition des marqueurs dans l'ensemble du côlon.

fréquemment (pas plus de 2 fois/jour) dans un côlon normal non préparé [10]. Chez l'enfant, il existe une diminution liée à l'âge de la fréquence des CPHA [11]. Lamplitude et la fréquence des contractions segmentaires ainsi que l'activité CPHA augmentent après un repas.

Outre des événements contractiles de faible importance, le côlon présente des variations du tonus musculaire qui augmente également après un repas et diminue pendant le sommeil [12]. Le rectum paraît également présenter un profil distinct de contraction, précédemment décrit comme «complexes moteurs rectaux» $[13,14]$, qui s'accroissent également après un repas.

\section{Défécation normale}

De façon sans doute surprenante, le profil des événements moteurs survenant au cours de la défécation n'a pas fait l'objet de nombreuses études. Lors d'une étude où une défécation avait été induite par l'introduction de bisacodyl dans le côlon gauche, des ondes péristaltiques d'amplitude élevée ont été produites et ont progressé vers la zone distale simultanément à une augmentation de la pression rectale. Le sphincter anal interne s'est relaxé simultanément au début du péristaltisme élevé dans le côlon gauche et est demeuré relâché jusqu'au moment où l'onde de pression a atteint l'anus et où la selle a été expulsée. Ces observations suggèrent que le profil moteur nécessaire pour la défécation se compose d'un péristaltisme colique, de contractions rectales et d'une relaxation anale précoce. Ce type coordonné d'activité motrice est similaire aux événements survenant dans l'œsophage lors du péristaltisme induit par la déglutition et associé à une relaxation précoce du sphincter inférieur de l'œsophage.

Au cours de la défécation, la quantité de contenu colique vidangé est variable et dépend de la zone colique où ont débuté les CPHA. Le côlon peut se vidanger à partir d'une zone très éloignée telle que le côlon transverse dis- 
tal ou d'une zone très en aval telle qu'uniquement le rectum. Quand la défécation est supprimée, il se produit des mouvements rétrogrades du contenu colique.

\section{Contrôle de la fonction motrice colique}

Le contrôle de la fonction motrice colique réside dans les nerfs extrinsèques (neurones corticaux et rachidiens), les nerfs intrinsèques coliques, les cellules interstitielles de Cajal qui agissent comme des régulateurs de rythme et dans les propriétés des cellules musculaires lisses du côlon elles-mêmes. L'innervation extrinsèque agit comme un activateur ou un inhibiteur des contractions coliques. Bien que la défécation soit placée sous contrôle cortical, un centre majeur de sa régulation est le pont [15]. Des études menées chez des patients atteints d'une affection de la moelle épinière ont suggéré que la voie du contrôle médullaire de la défécation se situait dans le cordon latéral à proximité immédiate des voies importantes pour le contrôle de la miction [16]. Les nerfs intrinsèques des plexus myentériques et sous-muqueux coordonnent les influx sensoriels provenant de la muqueuse colique et les afflux moteurs et peuvent être considérés comme contenant les "programmes» responsables des types d'activité contractile.

Une couche spécialisée de cellules régulant les rythmes, les cellules interstitielles de Cajal, est présente le long de la surface sous-muqueuse des muscles circulaires, dans les structures septales entre les faisceaux musculaires de ces derniers, et est associée aux neurones myentériques [17]. Ces cellules sont capitales pour la propagation des ondes lentes, l'organisation des contractions segmentaires et la fréquence des CPHA.

\section{Perturbation de la motricité colique dans la constipation}

\section{Études du transit}

Comme décrit plus haut, des marqueurs radio-opaques permettant de mesurer la durée totale du transit intestinal peuvent être employés afin de différencier une affection colique généralisée d'une obstruction. Dans la constipation fonctionnelle, les marqueurs apparaissent habituellement dans le rectum dans les 36 heures. Dans la constipation par transit lent ou les maladies neuromusculaires coliques, les marqueurs sont dispersés dans l'ensemble du côlon pendant de nombreux jours avec un temps total de transit intestinal excédant 84 heures. En ce cas, une biopsie sur la totalité de l'épaisseur ou une manométrie colique peut permettre de différencier une neuropathie d'une myopathie.

\section{Types de perturbations de la motricité}

Motricité colique

Les premières études de la motricité colique chez des patients constipés n'ont souvent porté que sur le côlon distal et ont été de courte durée. Elles ont démontré un accroissement de l'activité segmentaire.

Dans le cas d'une constipation idiopathique sévère, l'anomalie la plus importante de l'activité motrice colique est toutefois une diminution de la fréquence et de la durée des mouvements de masse. Une étude menée au moyen d'un tube manométrique inséré par coloscopie a porté sur les mouvements de masse chez 14 femmes présentant une constipation chronique [18]. Les patientes ont présenté en moyenne 2,6 mouvements de masse par 24 heures comparativement à une moyenne de 6,1 chez les témoins. Une absence de mouvement de masse pendant les 24 heures a été observée chez 4 patientes mais chez aucun des témoins. Les mouvements de masse ont été de plus courte durée chez les patientes, passant d'une moyenne de 14,1 à 8,2 secondes. Chez les patientes, ces mouvements ont donc progressé sur une moindre distance le long du côlon, car la vitesse de propagation a été la même dans les deux groupes $(1 \mathrm{~cm} / \mathrm{s})$.

La réponse colique à un repas est également altérée chez les patients souffrant de constipation sévère. La réponse colique à un repas standard a été étudiée chez 15 patients présentant une constipation par transit lent et 29 volontaires en bonne santé [9]. Après la prise d'un repas, l'activité contractile a été plus courte et les contractions propagées à amplitude élevée ont été moins nombreuses dans les côlons transverse, descendant et sigmoïde chez les patients que chez les sujets en bonne santé.

La fréquence des CPHA est plus faible chez les patients atteints d'une constipation idiopathique sévère, mais il apparaît que le programme neurologique régissant le péristaltisme et l'aptitude du côlon à la contraction est intact. Des CPHA fréquentes ont été induites au moyen d'un stimulant, le bisacodyl, lors d'une étude du péristaltisme colique chez des femmes atteintes de constipation sévère [19]. Les CPHA ont progressé vers la partie distale afin d'induire une contraction rectale, une relaxation anale et une évacuation efficace, similaires à celles survenant chez les sujets en bonne santé [20].

Dans la constipation fonctionnelle, la motricité colique est normale, avec présence de CPHA à la suite du réveil ou d'un repas, et l'indice de motricité augmente après 
une prise d'aliments. Une diminution de la fréquence des CPHA a été décrite chez des adultes présentant une inertie colique [9] et chez des enfants atteints d'une neuropathie impliquant le côlon [21]. Des études prolongées de la motricité colique couplées à des enregistrements de l'intestin grêle ont été utilisées afin de prévoir le succès d'une intervention chirurgicale chez des adultes atteints de constipation sévère $[22,23]$. La manométrie colique peut permettre d'identifier le segment du côlon où la fonction motrice est anormale et également de prendre plus facilement la décision de reconnecter un côlon dérivé. Le traitement de choix des nourrissons présentant des signes évoquant une pseudo-obstruction colique consiste à réaliser une iléostomie de décompression. Avec la croissance de l'enfant et l'amélioration des symptômes, la décision de reconnecter le côlon peut reposer sur les résultats d'études de la motricité du côlon dérivé et sur la connaissance de l'activité de l'affection neuromusculaire entérique sous-jacente [24, 25]. La manométrie colique est également utile pour la clarification du mécanisme d'une incontinence fécale chez un enfant ayant fait l'objet d'une intervention chirurgicale pour maladie de Hirschsprung et présentant une encoprésie malgré une fonction satisfaisante du sphincter anal. Des CPHA répétitives qui se propagent au néo-rectum immédiatement au-dessus des sphincters anaux (au lieu de s'arrêter au-dessus du plancher pelvien) peuvent vaincre la résistance des sphincters anaux interne et externe et produire une émission involontaire de selles.

Dans les affections neuromusculaires coliques, en l'absence de dilatation généralisée du côlon, les enfants dont les contractions ne s'accroissent pas après un repas et/ou qui ne produisent pas de CPHA peuvent être atteints d'une neuropathie colique ou d'une pathologie inflammatoire du côlon, qui affecte également le système neuromusculaire. Les enfants chez lesquels les contractions coliques sont totalement absentes sont plus probablement atteints d'une myopathie habituellement associée à un côlon dilaté.

\section{Motricité anorectale}

La manométrie anorectale est largement utilisée afin de diagnostiquer la maladie de Hirschsprung et d'étudier la constipation fonctionnelle. Dans la maladie de Hirschsprung, l'absence de réflexe recto-anal inhibiteur lors d'une distension rectale a été considérée comme diagnostique mais il existe un sous-groupe non négligeable d'enfants atteints d'une pseudo-maladie de Hirschsprung chez lesquels le réflexe recto-anal inhibiteur est anormal, ou même absent, démontrant que la manométrie anorec-

Physiopathologie de la constipation tale n'est pas spécifique de la maladie de Hirschsprung et que, pour un diagnostic définitif, une aspiration ou une biopsie rectale de la totalité de l'épaisseur est capitale. Les résultats des études de la constipation fonctionnelle sont divergents et il est souvent difficile de savoir si les anomalies manométriques représentent un trouble primitif ou sont secondaires à la rétention fécale chronique ou volontaire [26].

L'échographie anale et la défécographie n'ont été que rarement utilisées pour l'évaluation d'un dysfonctionnement des voies digestives basses chez l'enfant.

\section{Étiologie des anomalies de la motricité}

Les perturbations de la motricité colique et anorectale relèvent probablement de mécanismes différents dans les divers types de constipation. Un défaut de la contraction musculaire peut être présent chez les patients présentant un mégarectum ou un mégacôlon, mais ce trouble n'est dû à une atteinte organique affectant la neuromusculature colique que chez moins de $10 \%$ des enfants [1].

\section{Constipation fonctionnelle}

La constipation est d'origine fonctionnelle chez la majorité des enfants. Un nourrisson peut ne pas émettre de selles en raison d'apports hydriques ou alimentaires insuffisants. Tandis que le côlon conserve l'eau, les selles deviennent dures et difficiles à émettre et, en conséquence, la défécation est inhibée. Parfois, un nourrisson allaité au sein n'émet pas de selles pendant plusieurs jours en raison d'une importante diminution des résidus non absorbables. Dans ce cas, l'ajout de jus de fruits à l'alimentation et une augmentation des apports liquidiens sont suffisants pour ramollir les selles et augmenter la fréquence des exonérations.

Quand les parents réagissent excessivement aux efforts de défécation de l'enfant, leur anxiété associée à chaque tentative devient perturbante pour leur enfant. L'enfant peut répondre au besoin urgent de déféquer par des tentatives de rétention, en contractant le sphincter anal et les muscles fessiers. Le rectum s'adapte à la masse fécale et le besoin de déféquer disparaît. Avec le temps, ce comportement devient une réponse apprise automatique. L'émission d'une selle très volumineuse, qui distend douloureusement le sphincter anal, renforce la conviction que la défécation doit être évitée. Tandis que la paroi du rectum se dilate, le sphincter anal se relaxe de façon inadéquate et une encoprésie peut survenir, irritant les parents et effrayant l'enfant. Divers autres facteurs jouent également un rôle. Des tentatives d'apprentissage de l'utilisation des toilettes à un âge trop précoce $(<2,5$ ans) et 
une pression excessive afin d'obtenir des habitudes parfaites de défécation quotidienne sont à l'origine d'une intense lutte entre les parents et l'enfant, qui retient ses selles dans son rectum. L'entrée à l'école est une autre période où des difficultés de défécation peuvent également débuter en raison d'une angoisse de séparation et de l'état peu accueillant des toilettes scolaires. Les distractions que constituent les jeux avec des amis ou la télévision peuvent persuader l'enfant de retarder de façon répétée la défécation, induisant ainsi une constipation [2].

Troubles nerveux extrinsèques

Une affection neurologique, qu'elle affecte le cerveau ou l'innervation médullaire, pourrait être ensuite la cause la plus fréquente de troubles de la défécation. Le côlon paraît dépendre de son innervation extrinsèque pour le maintien d'une fonction normale, le côlon distal recevant quant à lui une innervation directe des racines S2-S4 [27]. Les affections neurologiques sont sous-estimées tant chez les adultes que chez les enfants. Une analyse de 31 millions de diagnostics à la sortie de l'hôpital chez des adultes âgés a montré que le groupe le plus important d'affections associées à une constipation se composait de maladies neurologiques et psychiatriques [28]. Des données similaires ne sont pas disponibles chez l'enfant, mais les pédiatres sont fréquemment confrontés à des problèmes de ce type. Ces enfants représentent une population très hétérogène, avec des mécanismes différents importants dans différents groupes d'enfants. L'absence de fibres alimentaires résulte en des selles dures chez les enfants alimentés par sonde. L'absence de tonus musculaire squelettique normal et de coordination résulte en de faibles efforts de défécation. Les médicaments fréquemment administrés aux enfants atteints d'une maladie neurologique, tels que les opiacés, les anticonvulsivants et les anticholinergiques, induisent une altération additionnelle de la motricité colique. Une étude menée chez des enfants atteints d'une infirmité motrice cérébrale a montré un retard du transit dans le côlon gauche chez ceux qui présentaient une constipation [29]. La motricité colique et rectale est modulée par des nerfs extrinsèques se composant de fibres nerveuses tant parasympathiques que sympathiques. Le côlon proximal reçoit une innervation cholinergique du nerf vague et le côlon distal des influx cholinergiques provenant des nerfs pelviens sacrés (racines S2-S4) [27]. Les nerfs splanchniques, avec des corps de neurone dans le ganglion mésentérique supérieur, fournissent une innervation adrénergique au côlon proximal et les nerfs lombaires, avec des corps de neurone dans les ganglions mésentériques inférieurs, assurent l'innerva- tion du côlon distal. Une lésion des nerfs extrinsèques, et donc une disparition des influx inhibiteurs parvenant à l'intestin, entraînent des anomalies de la motricité colique et anorectale. Chez l'enfant atteint d'une lésion de la moelle épinière ou d'un dysraphisme spinal, la réponse gastrocolique a disparu, le réflexe inhibiteur recto-anal est habituellement préservé mais le sphincter externe est souvent paralysé et la perception du besoin de déféquer peut disparaître. En conséquence, les enfants atteints d'un myéloméningocèle peuvent présenter à la fois une constipation et une incontinence fécale [30].

\section{Affections neuromusculaires intrinsèques}

Moins de $10 \%$ des enfants constipés sont atteints d'une affection congénitale ou acquise affectant le système neuromusculaire intestinal [1]. La plus fréquente des anomalies congénitales est la maladie de Hirschsprung, dont l'incidence est de 1 pour 4500 naissances vivantes contre 1 pour 7000 en ce qui concerne les malformations anorectales et 1 pour 40000 pour ce qui est des autres troubles neuromusculaires intestinaux. L'analyse des différentes et nombreuses maladies neuromusculaires excède le champ du présent article et les lecteurs intéressés sont invités à consulter une récente analyse de la littérature [31].

Les atteintes acquises sont presque toujours d'origine inflammatoire et peuvent se présenter sous la forme d'une constipation intraitable sévère ou d'épisodes récidivants de pseudo-obstruction [32, 33]. Des mécanismes autoimmuns sont souvent présents et répondent à un traitement immunosuppresseur [32, 34]. Dans d'autres cas, particulièrement chez les enfants atopiques, la constipation peut être secondaire à une allergie alimentaire [35].

Il est désormais reconnu que des altérations de l'environnement du système neuromusculaire, sous l'effet de troubles inflammatoires ou sous celui de l'environnement hormonal circulant, peuvent exercer un effet direct sur la fonction colique. Le rôle des hormones stéroïdiennes a été étudié chez des jeunes femmes atteintes d'une constipation idiopathique sévère [36]. Ces hormones ont été comparées entre les phases folliculaires et lutéales chez 23 femmes en bonne santé et 26 patientes atteintes d'une constipation idiopathique. Ces dernières ont constamment présenté une réduction de la concentration de la plupart des hormones stéroïdiennes aux deux phases du cycle mais aucune anomalie des hormones hypophysaires ou de la globuline liant les hormones sexuelles n’a été décelée. Aucune étude de ce type n’a été menée chez des adolescentes présentant une constipation, mais il n'existe aucune raison de penser que les résultats pourraient être différents.
Milla 
Des hormones gastro-intestinales circulantes ont été également étudiées chez des patients présentant une constipation idiopathique sévère par transit lent [37]. La réponse de ces hormones à un repas standard a été évaluée chez 12 femmes atteintes d'une constipation et 12 sujets témoins. La somatostatine a été élevée chez les femmes présentant une constipation, mais il n'a pas été possible de déterminer si cette anomalie était primaire ou secondaire.

\section{Références}

$>1$ Milla PJ: Endothelins, pseudo-obstruction and Hirschsprung's disease. Gut 1999;44: 148-149.

2 Hyman PE, Fleisher D: Functional fecal retention. Pract Gastroenterol 1992;16;29-37.

-3 Tucker DM, Sanstead HH, Logan GM, et al: Dietary fibre and personality factors as determinants of stool output. Gastroenterology 1981;81:879-883.

-4 Davies GJ, Crowder M, Reid B, Dickerson JWT: Bowel function measurements of individuals with different eating patterns. Gut 1986;27:164-169.

5 Metcalf AM, Phillips SF, Zinmeister AR, et al: Simplified assessment of segmental colonic transit. Gastroenterology 1987;92:4047.

-6 Narducci F, Bassotti G, Gaburri M, Morelli A: Twenty four hour manometric recording of colonic motor activity in healthy man. Gut 1987;28:969-973.

7 Cannon WB: The movements of the intestines studied by means of roentgen rays. Am J Physiol 1901;6:251-277.

8 Bassotti G, Imbimbo BP, Betti C, et al: Impaired colonic response to eating in patients with slow transit constipation. Am J Gastroenterol 1992;87:504-508.

-9 Bassotti G, Gaburri M: Manometric investigation of high amplitude propagated contractile activity of the human colon. Am J Physiol 1988;255;G660-G664.

$>10$ Crowell MD, Bassotti G, Cheskin LJ, et al: Method for prolonged ambulatory monitoring of high-amplitude propagated contractions from colon. Am J Physiol 1991;261: G263-G268.

11 Di Lorenzo CA, Flores F, Hyman PE: Agerelated changes in colon motility. J Pediatr 1995; 127:593-596.

12 Steadman CJ, Phillips SF, Camilleri M, et al: Variations of muscle tone in the human colon. Gastroenterology 1991;101:373-381.

-13 Kumar D, Williams NS, Waldron D, Wingate DL: Prolonged manometric recording of anorectal motor activity in ambulant human subjects: evidence of periodic activity. Gut 1989;30:1007-1011

14 Orkin BA, Hanson RB, Kelly KA: The rectal motor complex. J Gastrointest Motil 1989;1: $5-8$.
15 Weber J, Denis PL, Mihout B: Effect of brain stem lesion on colonic and anorectal motility. Dig Dis Sci 1985;30:419-425.

16 Nathan PW, Smith MC: Spinal pathways subserving defaecation and sensation from the lower bowel. J Neurol Neurosurg Psychiatry 1953;16:245-256.

17 Sanders KM, Stevens R, Burke E, Ward SM: Slow waves actively propagate at submucosal surface of circular layer in canine colon. Am J Physiol 1990;259:G258-G263.

18 Bassotti G, Gaburri M, Imbimbo BP, et al: Colonic mass movements in idiopathic chronic constipation. Gut 1988;29:11731179.

19 Kamm MA, van der Sijp JRM, LennardJones JE: Observations on the characteristics of stimulated defaecation in severe idiopathic constipation. Int J Colorect Dis 1992;7: 197-201.

20 Kamm MA, van der Sijp J, Lennard-Jones JE: Colorectal and anal motility during defaecation. Lancet 1992;339:820.

21 Di Lorenzo C, Flores FA, Reddy SN, et al: Colon motility in symptomatic children after surgery for Hirschsprung's disease: abnormalities in 'healthy' colon. Gastroenterology 1991;100:A437.

22 Bassotti G, Betti C, Felli MA, et al: Extensive investigation of colonic motility with pharmacological testing is useful for selecting surgical options in patients with inertia colica. Am J Gastroenterol 1992;87:143-147.

23 Redmond JM, Smith GW, Barofsky I, et al: Physiological tests to predict long-term outcome of total abdominal colectomy for intractable constipation. Am J Gastroenterol 1995;90:748-753.

24 Heneyke S, Smith VV, Spitz L, Milla PJ: Chronicintestinal pseudo-obstruction: treatment and long term follow up of 44 patients. Arch Dis Child 1999;81:21-27.

25 Ordein JJ, Di Lorenzo C, Flores AE, et al: Diversion colitis in children with severe gastrointestinal motility disorders. Am J Gastroenterol 1992;87:88-90.
26 Meunier P, Marechal JM, de Beaugeu MJ: Rectoanal pressures and rectal sensitivity studies in chronic childhood constipation. Gastroenterology 1979;77:330-336.

27 Varma JS, Binnie N, Smith AN, et al: Differential effects of sacral anterior root stimulation on anal sphincter and colorectal motility in spinally injured man. Br J Surg 1986;73: 478-482.

28 Johanson JF, Sonnenberg A, Koch TR, et al: Association of constipation with neurologic diseases. Dig Dis Sci 1992;37:179-186.

29 Staiano AM, Del Giudice E: Colonic transit and anorectal manometry in children with severe brain damage. Pediatrics 1994;94: 169-173.

30 Wald A: Use of biofeedback in fecal incontinence in patients with meningomyelocele. Pediatrics 1981;68:45-49.

31 Smith VV, Milla PJ: Developmental disorders; in Spiller R, Grundy SD (eds): Pathophysiology of the Enteric Nervous System: A Basis for Understanding Functional Diseases. Oxford, Blackwell, 2004, pp 47-60.

32 Smith VV, Gregson N, Foggensteiner L, et al: Acquired intestinal aganglionosis and circulating autoantibodies without neoplasia or other neural involvement. Gastroenterology 1997;112:1366-1371.

33 Schappi MG, Smith VV, Milla PJ, Lindley KJ Eosinophilic myenteric ganglionitis is associated with functional intestinal obstruction. Gut 2003;52:752-755.

34 Ruuska TH, Karikoski R, Smith VV, Milla PJ: Acquired myopathic intestinal pseudoobstruction may be due to autoimmune enteric leiomyositis. Gastroenterology 2002; 122:1133-1139.

35 Shah N, Lindley K, Milla P: Cow's milk and chronic constipation in children. N Engl J Med 1999;18:340:891-892.

-36 Kamm MA, Farthing MJG, Lennard-Jones JE, et al: Steroid hormone abnormalities in women with severe idiopathic constipation. Gut 1991;32:80-84.

37 van der Sijp JRM, Kamm MA, Nightingale $\mathrm{JM}$, et al: Abnormal circulating somatostatin and pancreatic glucagon levels in patients with severe idiopathic constipation. Gastroenterology 1993;104:A593. 